\title{
La actividad física disminuye el riesgo de fracturas osteoporóticas en mujeres mayores
}

Study of osteoporotic fractures research group. Gregg E, Cauley J, Seeley D et al. Ann.Intern. Med. 1998; 129:81-88.

\section{Objetivo}

Determinar si mayores niveles de actividad física se asocian con menor incidencia de fracturas de muñeca, cadera y columna vertebral, en mujeres mayores.

\section{Diseño}

Estudio multicéntrico prospectivo de cohortes.

\section{Lugar}

Cuatro centros de Estados Unidos.

\section{Pacientes}

Participaron 9704 mujeres mayores de 65 años enroladas previamente en el Study of 0steoporotic Fractures Group, exceptuándose mujeres de raza negra, institucionalizadas, con reemplazo bilateral de cadera o incapaces de caminar.

\section{Evaluación de factores pronósticos}

Se evaluó peso, estatura, densidad mineral ósea en radio y calcáneo, fuerza muscular; autovaloración del estado de salud, ingesta de calcio, alcohol, uso de terapia de reemplazo hormonal (TRH), benzodiazepinas u otros ansiolíticos, y capacidad para caminar, subir escaleras, y desarrollar tareas domésticas. Se evaluó también la actividad física rea lizada en el último año: número de cuadras caminadas por día, pisos subidos por escalera, tareas domésticas y jardinería. La inactividad se estimó según el número de horas diarias que pasaban sentadas. Se las clasificaron en quintilos de actividad física creciente.

\section{Medición del resultado principal}

Se evaluó el riesgo de fractura según quintilos de actividad física total (medida en $\mathrm{Kcal} / \mathrm{semana}$ y calculada a través de un cuestionario al inicio del estudio). También se evaluó el riesgo según la intensidad de actividad física: inactivas; baja; y moderada o alta intensidad. La incidencia de fracturas vertebrales se documentó comparando las radiografías de columna obtenidas al inicio del estudio con las del seguimiento (en promedio 3.7 años después). El resto de las fracturas se determinó con un se- guimiento telefónico cada 4 meses, y confirmación con radiografías (media de seguimiento 7.6 años). Las variables que se tuvieron en cuenta para el análisis multivariable* fueron: edad, peso, tabaquismo, TRH, ingesta de calcio, alcohol, caídas y percepción del propio estado de salud.

\section{Resultados principales}

Las mujeres en el menor quintilo de actividad física tenían mayor edad y peso, eran más fumadoras, consumían menos alcohol y calcio y recibían menos estrógenos que las mujeres en el mayor quintilo $(p<0.05)$; en este primer grupo se registró una mayor incidencia de caídas. Las mujeres más activas reportaban menos dificultades funcionales, mejor salud y mayor densidad mineral ósea que las mujeres menos activas $(p<0.001)$. Con respecto a las fracturas de cadera, la incidencia fue de 5.8 por 1000 , la cual se reducía a medida que avanzaban los quintilos de actividad física, teniendo las del $5^{\circ}$ vs. $1^{\circ}$ quintilo un $42 \%$ menos de riesgo de fractura ajustado por edad ( $R R=0.58$, IC95\% 0.42-0.80). A mayor intensidad de actividad física, menor incidencia de fracturas, siendo la reducción del riesgo ajustada por la edad de $27 \%$ para la de baja intensidad, y $45 \%$ para la de moderada a vigorosa en comparación con las inactivas. En cuanto a las tareas domésticas pesadas, la reducción fue del $22 \%$ si era $>10 \mathrm{hs} /$ semana (IC95\% $2 \%$ a $39 \%$ ). Las mujeres que pasan sentadas más de 9 hs/día tienen un aumento de riesgo de $43 \%$ (IC95\% 12\% a 82\%). Luego del ajuste de variables como tabaquismo, TRH, peso, ingesta de calcio y otras, las mujeres de los dos quintilos superiores presentaron una reducción en el riesgo de fractura ( $R R=$ 0.64, IC95\% 0.45-0.89, $\mathrm{p}<0.001$ ). Para las fracturas de muñeca, si bien las mujeres con mayor actividad física tuvieron menor riesgo, éste no fue estadísticamente singificativo. En la fracturas vertebrales, la actividad física total no modificó el riesgo de fractura; pero las que realizaban actividades más intensas tuvieron menor riesgo.

\section{Conclusiones}

Las actividad física de moderada a vigorosa se asocia a menor riesgo de fractura de cadera pero no altera el de fractura de muñeca o vertebral.

\section{COMENTARIO}

La osteoporosis es una de las mayores causas de morbimortalidad y de gastos médicos, padeciéndola 1 de cada 3 mujeres postmenopáusicas y la mayoría de los ancianos ${ }^{1}$. La fractura de cadera es la más devastadora de las fracturas osteoporóticas, ya que durante el primer año de ocurrida, tiene una mortalidad del $20 \%$, y por lo menos la mitad de los sobrevivientes necesitan ayuda para desarrollar las actividades de la vida diaria 1-2. Durante años se postuló la actividad física como forma de prevención de la osteoporosis, ya que actuaría favorablemente en la remodelación del hueso.

Uno de los elementos que refuerza el hallazgo de la protección de la fractura de cadera con el ejercicio, es la relación dosis respuesta, pues aquellas mujeres con actividad física moderada a vigorosa tienen mayor reducción que con actividades bajas. Sin embargo, pareciera que el efecto protector de la misma estaría dado si se desarrolló con frecuencia durante la juventud y adultez, como así también en mujeres moderadamente activas; pero se observó que en mujeres muy activas recientemente (en la postmenopausia) no ejercería un efecto protector para la reducción de la fractura de cadera ${ }^{4}$. Este estudio, junto a otros similares, ${ }^{3-4}$ concluyen que la actividad física reciente aumentaría el riesgo de fractura de cadera. Esto se debe a que en mujeres inactivas durante el pasado, una actividad vigorosa podría acrecentar las probabilidades de caída, y ejercería demasiado stress en huesos realmente débiles, dando así una mayor probabilidad de fracturas. De todas maneras, hay que considerar que al desarrollar una actividad física, no sólo se incrementa la masa ósea sino también se mejora la fuerza muscular, la coor- dinación y balanceo, y el tiempo de reacción frente a una eventual caída. Un ejemplo de ello es que las mujeres más ágiles extiendan sus manos frente a una caída, absorbiendo el impacto y el golpe y la consiguiente posible fractura de muñeca. Debido a que la actividad física tardía no aumenta suficientemente la densidad ósea, no se observa relación con la disminución de fracturas vertebrales. En cuanto a las observaciones al presente estudio, parece generalizable a nuestra población predominantemente blanca, sin dejar de lado que los voluntarios de un ensayo clínico por lo general son más saludables que la población general. En lo que respecta a la medición de la actividad física, sólo la evalúan una vez (por lo que no se pueden sacar conclusiones sobre los cambios del patrón de actividad fisica con el tiempo y la incidencia de fracturas) e interrogan acerca de la actividad física desarrollada en el año previo (por lo que no se puede saber la actividad física de esta población mayor durante la premenopausia ni su efecto a largo plazo). Aunque la muestra de las pacientes con fractura vertebral fue reducida debido a problemas para obtener las placas, la pérdida en el seguimiento o muerte, ésta es la primera evaluación prospectiva de la relación entre la actividad física y las fracturas vertebrales.

Con todo lo expuesto parecería más prudente estimular a nuestras pacientes a practicar una actividad física reglada durante toda la vida o en su defecto iniciarla en la postmenopausia. Esta actividad debería ser de moderada intensidad, aunque en las mujeres mayores y sedentarias parecería más prudente recomendar actividades de baja intensidad. 